\section{Misconception of consumers and retailers regarding fungal spoilage of onion and prevalence of fungi in rotten onion}

\author{
Md. Rafiqul Islam, Masud Prince', Syed Mohammad Lokman', Lolo Wal Marzan', \\ Shahidul Alam²
}

\author{
${ }^{1}$ Department of Genetic Engineering and Biotechnology, University of Chittagong, Chattagram, \\ Bangladesh \\ ${ }^{2}$ Department of Botany, University of Rajshahi, Rajshahi-6205, Bangladesh
}

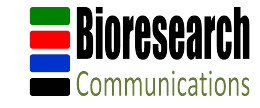

Bioresearch Communications

Volume 8, Issue 1, January 2022

DOI:

https://doi.org/10.3329/brc.v8i1.57046

\begin{abstract}
Onion is an inevitable part of our food habits. Fungal infection is one of the major reasons of onion spoilage which not only cause economic losses but also may cause public health threat through mycoses and mycotoxicoses. A total 15 onion samples from different places in Chattagram, Bangladesh were collected to assess the fungal contaminants.Onion consumers along with retailers were interviewed to evaluate their awareness about fungal food spoilage and associated health risk. Three different fungal species were identified and isolated by analysing their macroscopic and microscopic features. These isolates were Aspergillus niger, Aspergillus flavus, and Penicillium spp. A. niger was the most prevalent and found in 14 samples out of 15. Biochemical characterization of the isolated fungi was also done to assess their ability to produce extracellular enzymes and amylase, protease, and cellulase activities were observed. Survey data showed that only around $20 \%$ of the interviewees had some idea about fungal contamination, while nearly about $80 \%$ of them believed that washing, sunburn and cooking can make the food safe.
\end{abstract}

KEYWORDS: Fungal Spoilage, Food Safety, Onion Spoilage, Survey

RECEIVED: 12 August 2021, ACCEPTED: 30 October 2021

TYPE: Original Research
CORRESPONDING AUTHOR: Md. Rafiqul Islam, Department of Genetic Engineering and Biotechnology, University of Chittagong, Chittagong-4331 Email: rafiq.geb@cu.ac.bd

\section{Introduction}

Onion (Allium cepa $L$.) is widely cultivated vegetable in the world. There are various uses of onions, including culinary (vegetable, spice, and food favor), medicinal, and pharmacological. The all-year-long consumption of onion makes it an economically important vegetable ${ }^{1}$. Onion storage is done at different stages from farmer to consumer to ensure market demand. However, inexperienced handling and inadequate storage facilities can lead to large amounts of onion loss every year. One of the major causes for the reduction in onion yields is the various fungal infections during long-term storage which could lead up to $40 \%$ loss in developing countries. Different fungal species such as Penicillium sp., Aspergillus niger, Botrytis aclada, and Fusarium sp. are reported to cause blue mold, black mold, neck rot, and basal rot diseases in onions ${ }^{2-4}$.

Fungal spoilage not only causes severe damage to crop and vegetable production yield in terms of quality and quantity but also poses a threat to human health by being the major contributors to opportunistic infections ${ }^{5}$. Fungal infections in humans appear to be increasing due to the rise in immunocompromised patients worldwide. Exposure to spores of pathogenic fungi can lead to subclinical or unrecognized syndromes and allergic manifestations. However, Fungi are still not well-recognized as major pathogens by the public and public health authorities ${ }^{6}$.

Moreover, food stuffs are rich sources of nutrients that also facilitate fungal colonization. Interestingly and unfortunately for human and animal being when fungi grow on the foods and vegetables, they also produce toxins (mycotoxins) as their secondary metabolites. Mycotoxins are very harmful for human and animal health causing a wide range systemic disease and even may cause death ${ }^{7}$. A study on the Bangladeshi rural and urban population found the presence of aflatoxin, ochratoxin and citrinine in their urine indicating that people are spontaneously exposed to mycotoxin unconsciously ${ }^{8,9}$.

Above all food contamination and unsafe food consumption is a severe problem in Bangladesh ${ }^{10}$. Although Bangladesh is the $9^{\text {th }}$ onion producing country in the world, every year we need to import tons of onion ${ }^{11}$ because poor harvesting practices, storage and handling errors, commercial mentality, and lack of awareness contribute to the fungal spoilage leading to huge loss. Besides, the geographical location of Bangladesh (tropical country) gives it an optimum climatic condition (temperature, humidity) for fungal growth.

Onions are serves as cooked vegetables or spices (e.g., stirfried, dried, or roasted) or raw (flavor salads) foodis a source of carbohydrate, protein, vitamins, minerals and flavonoids ${ }^{1}$. In both ways, fungal-spoiled onions can mediate exposure to fungal spores and toxin through inhalation, dermal contact and/or ingestion. On the other hand, onion is a crucial part of our daily life food habit. People of Bangladesh cannot imagine their cuisine without onion even a single day. 
Authors necessitate to assess the people (retailers and consumers) awareness regarding fungal spoilage of onion and the consequences of such fungi on their health. In addition, we focus on the isolation, identification and biochemical characterization of fungi of spoiledonions collected from the local markets of Chattagram, Bangladesh.

\section{Materials and Methods}

\section{Study design, area, and population}

A semi-open-ended questionnaire was developed and a randomized cross sectional survey study was conducted to determine the level of public awareness about food safety in terms of fungal contamination of onion in the Chattagram Metropolitan area, University of Chittagong and Hathazari, Chattagram, Bangladesh ${ }^{12}$. Chattagram, the second-largest city in Bangladesh with a population of around 7.9 million, is the main port city of the country where almost all kinds of food items are imported from all over the world and then distributed to other parts of the country. Two sets of semiopen questionnaires were developed targeting two different stakeholders of the food supply chain: Retailers and consumers. 200 consumers and 50 retailers were interviewed personally with their consent. Both sets of questionnaires contain questions related to demographics (age, sex, educational qualifications, and economic status) as well as questions related to an individual's knowledge, attitude, and behavior towards food safety in terms of fungal food contamination. Special emphasis was given on questions related to onion consumption, fungal contamination of onion and people's awareness regarding the potential health hazards caused by such fungal spoilage of the food. Along with the survey data collection, onion samples were collected from the retailers interviewed for laboratory analysis and study was followed as Figure 1.

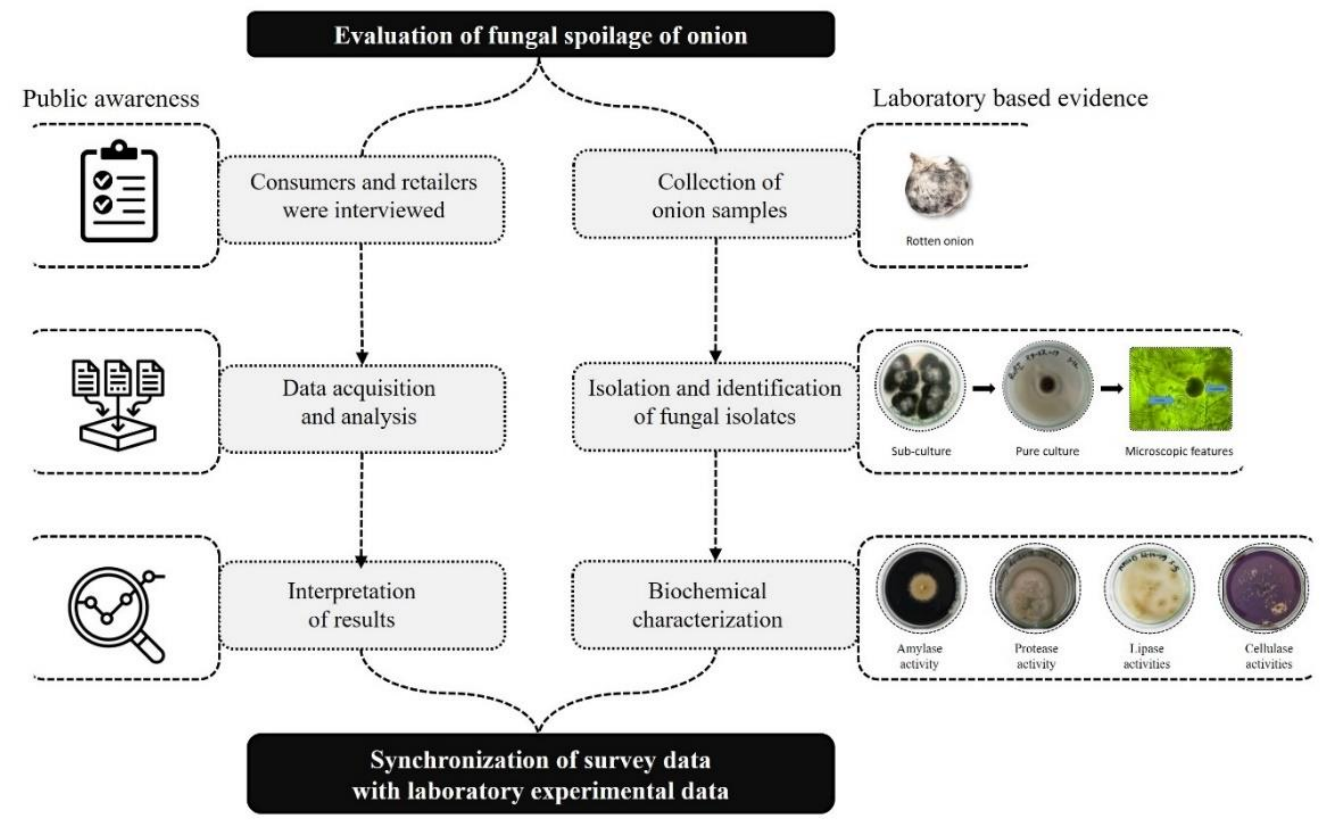

Figure 1. Schematic representation of study procedure.

\section{Sample collection and isolation of single fungal spore}

A total of 15 onion samples were collected from the retailers, immediately locked into sterile zip lock bags, brought to the laboratory in sterile condition and stored at $4^{\circ} \mathrm{C}$ until laboratory experiments. Onion samples were selected on the basis of appearances of fungal infections. Dry scales of the onion bulb tissues were stripped off and submerged into $70 \%$ ethanol for three minutes followed by a $1 \%$ sodium hypochlorite solution for two minutes and then washed three times with sterile distilled water. Rinsed samples were dried with sterile blotting paper. Small segments of tissues (3 cubic millimeters) from the margins of the rotted lesions were cut out with a sterile scalpel and placed on Petri plates containing PDA (potato dextrose agar) media. Plates were then incubated at $30^{\circ} \mathrm{C}$ for 5 days and fungal colonies were selected for subculture. The distinction of fungi were made on the basis of colony characters such as color, shape, elevation, etc. Single spores of each type were isolated using sterile toothpicks and subcultured on PDA (Potato dextrose agar) and YES (yeast extract, sucrose agar) media. Plating was done by spotting with the sterile toothpick in the center of the solid media surface. Afterward, plates were incubated at $30^{\circ} \mathrm{C}$ for 5-7 days and a modified protocol was followed ${ }^{13}$.

\section{Morphological study}

Macroscopic analyses of the isolated fungal colonies were done in terms of form, elevation, surface, opacity, and colors. To determine the microscopic features of the isolated fungi, microscopic slides were prepared and observed under an inverted microscope (OPTIKA XDS-2). The microscope was capable of 40X magnification and connected with a computer for capturing photos. The whole slide was then scanned visually to identify conidia size, shape, the structure of mycelia, and the way of spore distribution ${ }^{14}$.

\section{Biochemical characterization}

Onion contains carbohydrates, protein, and a low amount of lipid content. Hence, it is usual that they might express amylase, protease, and a little of lipase activity. During the growth of molds on the foods, they also might secrete 
cellulose degrading enzymes facilitating diffusing fungal spores deeper into the onion scales.

\section{Amylase, Protease, and Lipase activity test}

Plate assay method was applied using minimal media supplemented with $1 \%$ starch and $1 \%$ gelatin respectively for testing the carbohydrate degrading ability and the protease activity of the fungal isolates. To assess the lipase activity, fungal isolates were inoculated with tween 80 agar media. In every Petri plate, a small portion of the fungal isolates was spot inoculated and then incubated at $30^{\circ} \mathrm{C}$ for $5-7$ days. The incubated plates for testing amylase activity were flooded with iodine reagent and the plates for testing protease activity were flooded with aqueous solution of mercuric chloride reagent. The presence of the clear zone, visible precipitation around the fungal colony due to the effect of reagents indicates the presence of extracellular amylase and protease activity ${ }^{15}$.

\section{Cellulase activity test}

To determine the level of cellulase activity of the fungal isolates, they were inoculated in media containing cellulose (we used Carboxymethyl Cellulose) as substrate. The $\mathrm{pH}$ of the medium was adjusted at 5.5 , sterilized at $121^{\circ} \mathrm{C}$ and $15 \mathrm{psi}$ for 15 minutes. The media was transferred into Petri plates and fungal inoculation was done by spotting at the center of the plates using sterile toothpicks for 3 days of incubation at $27^{\circ} \mathrm{C}$. After the incubation period, the plates were flooded with $1 \%$ freshly prepared congo red solution and the plates were allowed to stand for 20 minutes at room temperature. Plates were then destained with $1 \mathrm{M} \mathrm{NaCl}$ solution. $5 \%$ acetic acid was then added to the plates for 2 minutes. Formation of a bluish-pink color of clear zone around the fungal colony indicates the presence of extracellular cellulase activity ${ }^{16}$.

\section{Results}

Food safety awareness among the consumers and retailers Socio-demographic status

Two-hundred consumers and fifty retailers were interviewed to assess their level of awareness about food safety. Representative sampleswere included to get a clear view of the level of public awareness about food safety and fungal spoilage of onion. After primary screening,15 consumer questionnaires were discarded because of incomplete submission. The interviewees were distributed mostly between $30-50$ years' age group (62\%) for retailers and 20-40 years' age group $(66 \%)$ for consumers (Table. 1$)$.

Table 1. Demographic characteristics of participants and the proportion of the participants.

\begin{tabular}{|c|c|c|}
\hline Features & $\begin{array}{l}\frac{\text { Retailers }}{N=50} \\
n(\%)\end{array}$ & $\begin{array}{l}\text { Consumers } \\
N=185 \\
n(\%)\end{array}$ \\
\hline \multicolumn{3}{|l|}{ A. Age limit } \\
\hline$>20$ & $1(2 \%)$ & $9(4.865 \%)$ \\
\hline $20-29$ & $9(18 \%)$ & $66(35.68 \%)$ \\
\hline $30-39$ & $17(34 \%)$ & $59(31.89 \%)$ \\
\hline $40-49$ & $14(28 \%)$ & $29(15.68 \%)$ \\
\hline$>50$ & $9(18 \%)$ & $22(11.89 \%)$ \\
\hline \multicolumn{3}{|l|}{ B. Marital status } \\
\hline Married & $45(90 \%)$ & $127(68.65 \%)$ \\
\hline Single & $5(10 \%)$ & $58(31.35 \%)$ \\
\hline \multicolumn{3}{|l|}{ C. Gender } \\
\hline Male & $50(100 \%)$ & $168(90.81 \%)$ \\
\hline Female & - & $17(9.189 \%)$ \\
\hline \multicolumn{3}{|c|}{ D.Educational qualifications } \\
\hline Master Degree & - & $15(8.108 \%)$ \\
\hline Bachelor Degree & $6(12 \%)$ & $67(36.22 \%)$ \\
\hline Diploma & - & $3(1.622 \%)$ \\
\hline HSC & $10(20 \%)$ & $26(14.05 \%)$ \\
\hline SSC & $17(34 \%)$ & $38(20.54 \%)$ \\
\hline Primary & $16(32 \%)$ & $34(18.38 \%)$ \\
\hline N/A & $1(2 \%)$ & $2(1.081 \%)$ \\
\hline \multicolumn{3}{|c|}{ E. Family member (person) } \\
\hline $1-2$ & $1(2 \%)$ & $0(0 \%)$ \\
\hline
\end{tabular}




\begin{tabular}{|l|l|l|}
\hline Features & $\begin{array}{l}\text { Retailers } \\
\text { N= 50 } \\
n(\%)\end{array}$ & $\begin{array}{l}\text { Consumers } \\
\boldsymbol{N}=\mathbf{1 8 5} \\
\boldsymbol{n}(\boldsymbol{\%})\end{array}$ \\
\hline E. Family member (person) & $16(32 \%)$ & $53(28.65 \%)$ \\
\hline $3-4$ & $23(46 \%)$ & $84(45.41 \%)$ \\
\hline $5-6$ & $10(20 \%)$ & $48(25.95 \%)$ \\
\hline$>6$ & & $13(7.027 \%)$ \\
\hline F. Income (Taka) & $2(4 \%)$ & $60(32.43 \%)$ \\
\hline$<11000$ & $21(42 \%)$ & $66(35.68 \%)$ \\
\hline $11000-20000$ & $17(34 \%)$ & $38(20.54 \%)$ \\
\hline $21000-30000$ & $10(20 \%)$ & $2(1.081 \%)$ \\
\hline $31000-50000$ & - & $6(3.243 \%)$ \\
\hline$>50000$ & - & \\
\hline N/A & & \\
\hline
\end{tabular}

$90 \%$ retailers were married while the portion of married consumers was $68.65 \%$. Most of the interviewees were male (100\% retailer and $90.81 \%$ consumers). Though the education level of most of the consumers were high $(44.3 \%$ completed graduation, $14 \%$ completed higher secondary level, and $20.5 \%$ completed secondary level), only $12 \%$ retailers hold a bachelor degree. More than $60 \%$ retailers and consumers have at least 5 family members. In terms of monthly income, $42 \%$ retailer had a monthly income of 11000-20000 taka per month while $34 \%$ and $20 \%$ had monthly income between 21000 30000 and 31000-50000 taka per month, respectively. On the other hand, the monthly income of about $32 \%, 35 \%$, and $20 \%$ consumers were between 11000-20000, 21000-30000, and 31000-50000 taka per month respectively (Table. 1).

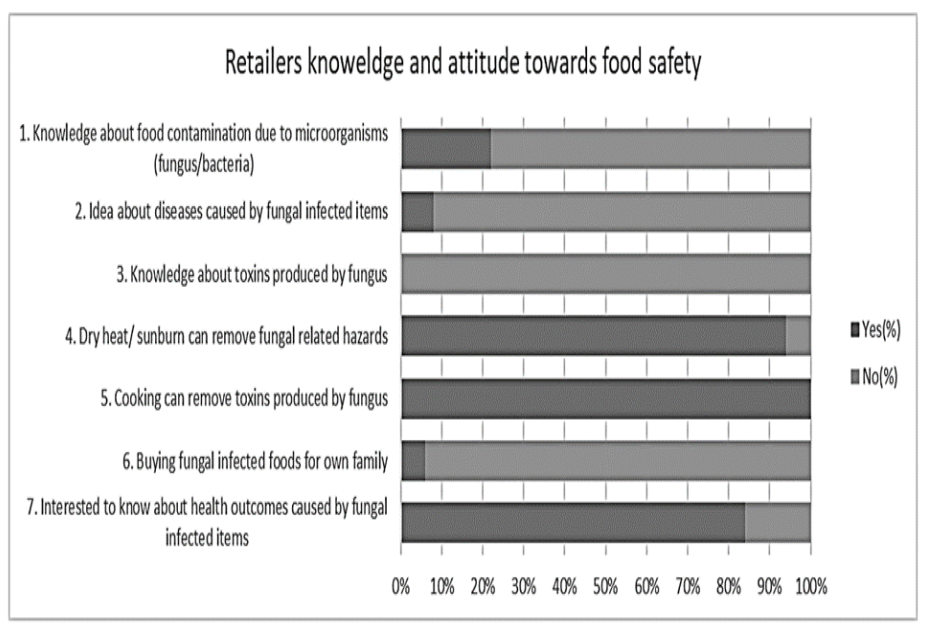

Knowledge, attitude and behavior of consumers and retailers towards food safety

The consumers were asked regarding the knowledge about the production of toxins from fungal spores. $23.78 \%$ consumers showed positive attitude while $76.22 \%$ responded as of having no idea about toxin produced from fungal spore. However, majority of them believe that either washing $(64.32 \%$ consumers), dry heating (54.05\% consumers), or cooking at high temperature $(76.76 \%$ consumers) could destroy the microorganism or the toxin. $38.9 \%$ consumers responded as having no idea about fungal contamination of onions while the rest $(61.1 \%)$ had positive attitude towards this issue. $82.7 \%$ interviewees expressed their concern about the safety and hygiene of the onion while $17.3 \%$ still don't take it as a concerning issue (Figure 2).

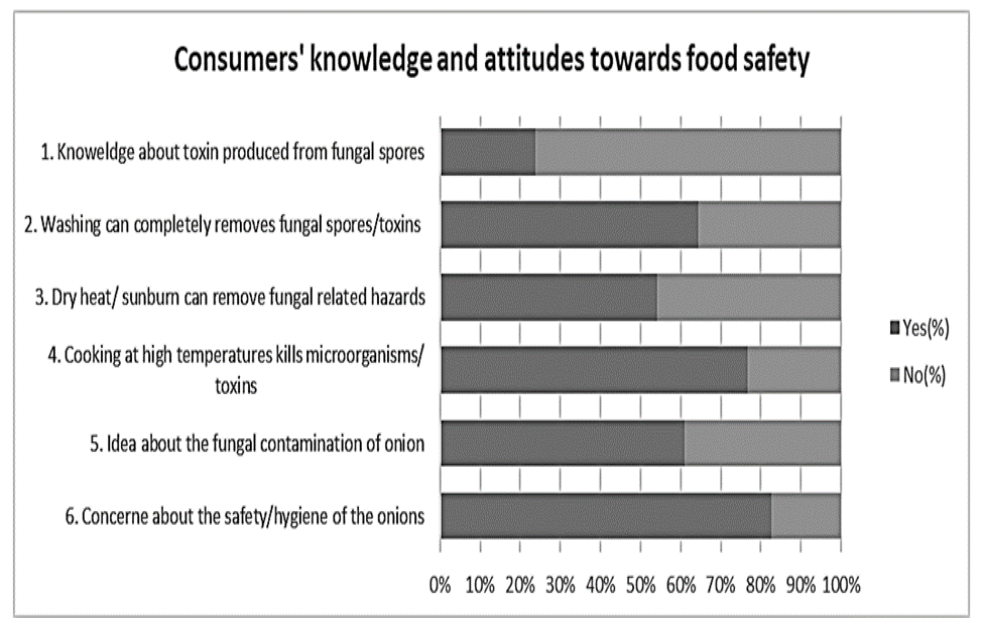

Figure 2. Knowledge and attitude of retailers and consumers towards food safety and fungal spoilage. 
50 grocery retailers were asked about their knowledge of food spoilage, and attitude towards food safety. Interview data showed that $78 \%$ of retailers did not have any previous knowledge about food contamination due to microorganisms and $92 \%$ retailers had no idea that fungal infected items could cause diseases. No retailers had heard anything about the production of toxin by fungi. Almost all the retailers believe that dry heat or cooking can remove fungal related hazards but $94 \%$ of them were not intended to buy such food items for their own family. However, $84 \%$ retailers expressed their interest to know the health outcomes caused by fungal infected items (Figure 2).

\section{Isolation and identification of fungal contaminants of onion samples}

15 onion samples were collected from retailers during conducting the survey and samples were cultured on PDA medium, where abundant growth of fungi was observed in 14 samples except sample 6 (Figure 3).

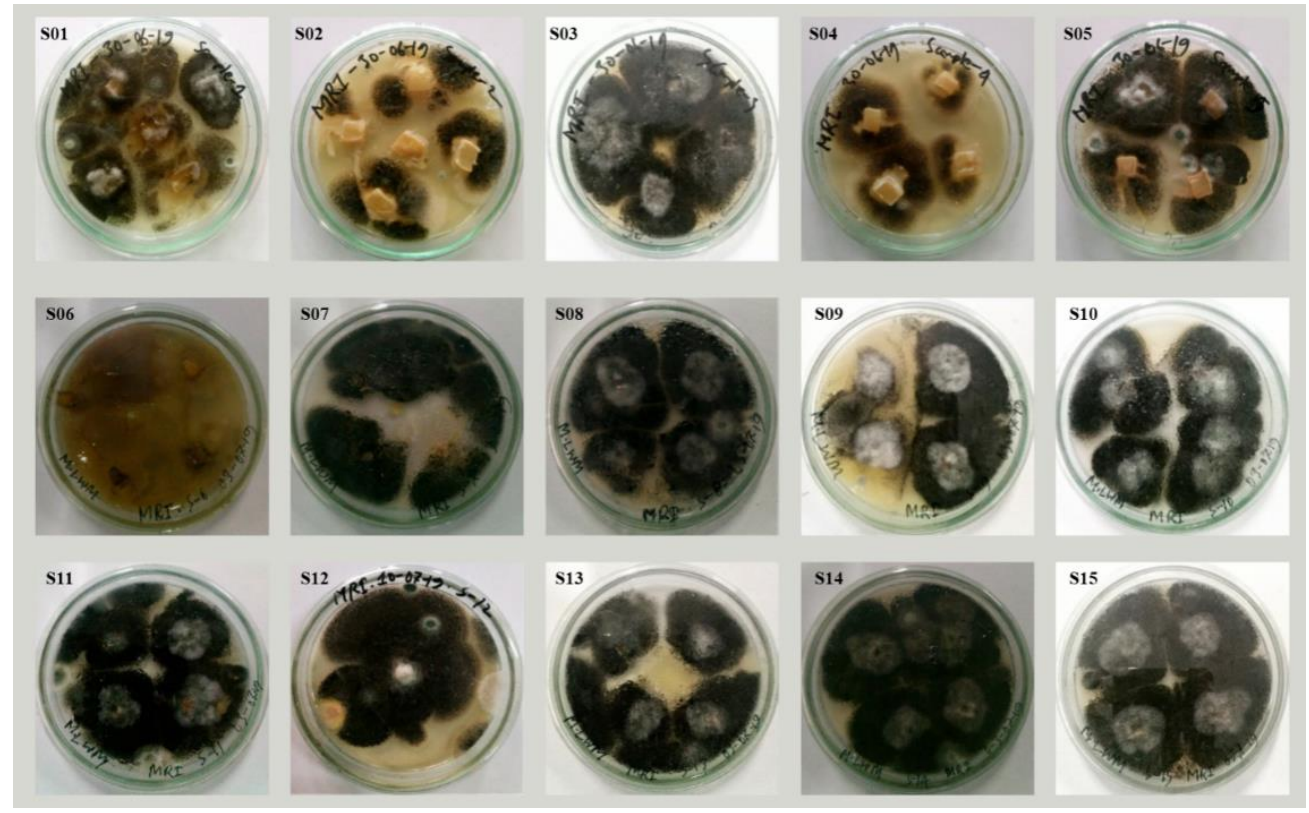

Figure 3. Fungal growth on Petri plates containing PDA media

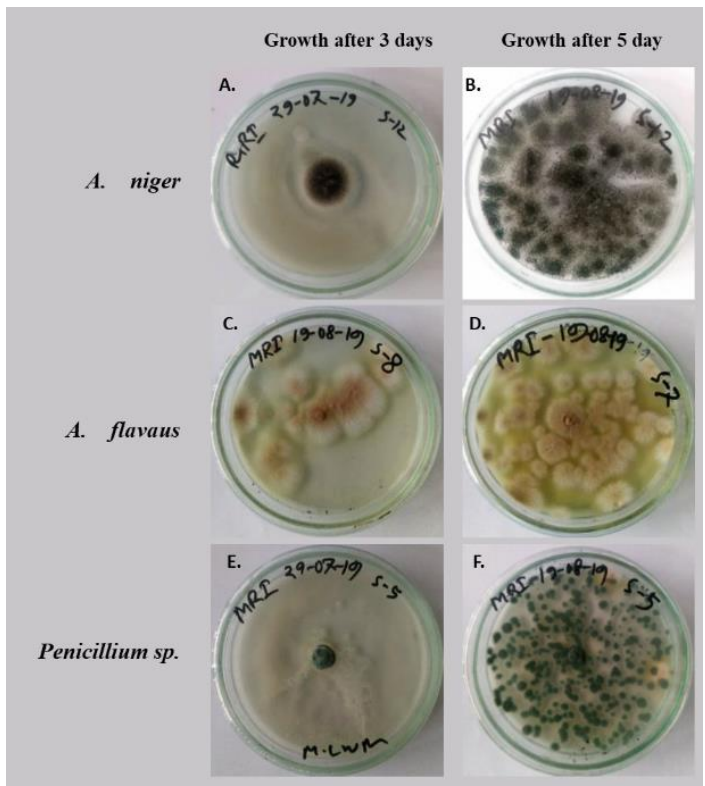

Figure 4. Pure culture and morphological features of fungal isolates. (A) Aspergillus niger growth after 3 days; (B): Aspergillus niger growth after 5 days; (C) Aspergillus flavaus growth after 3 days (D): Aspergillus flavus growth after 5 days; (E) Penicillium sp. growth after 3 days;(F): Penicillium sp. growth after 5 days. 
Pure culture of each isolated fungal species was subjected to morphological, macroscopic and microscopic analysis. All the isolates were identified based on their distinct macroscopic (Figure 4) and microscopic (Figure 5) characteristics according to previous literature reviews ${ }^{14,17-19}$ and 3 different fungal species- Aspergillus niger, Aspergillus flavus and Penicillium spp. were identified. Among the fungal species observed, Aspergillus niger was the most prevalent as 14 samples were contaminated with it (Table 2).
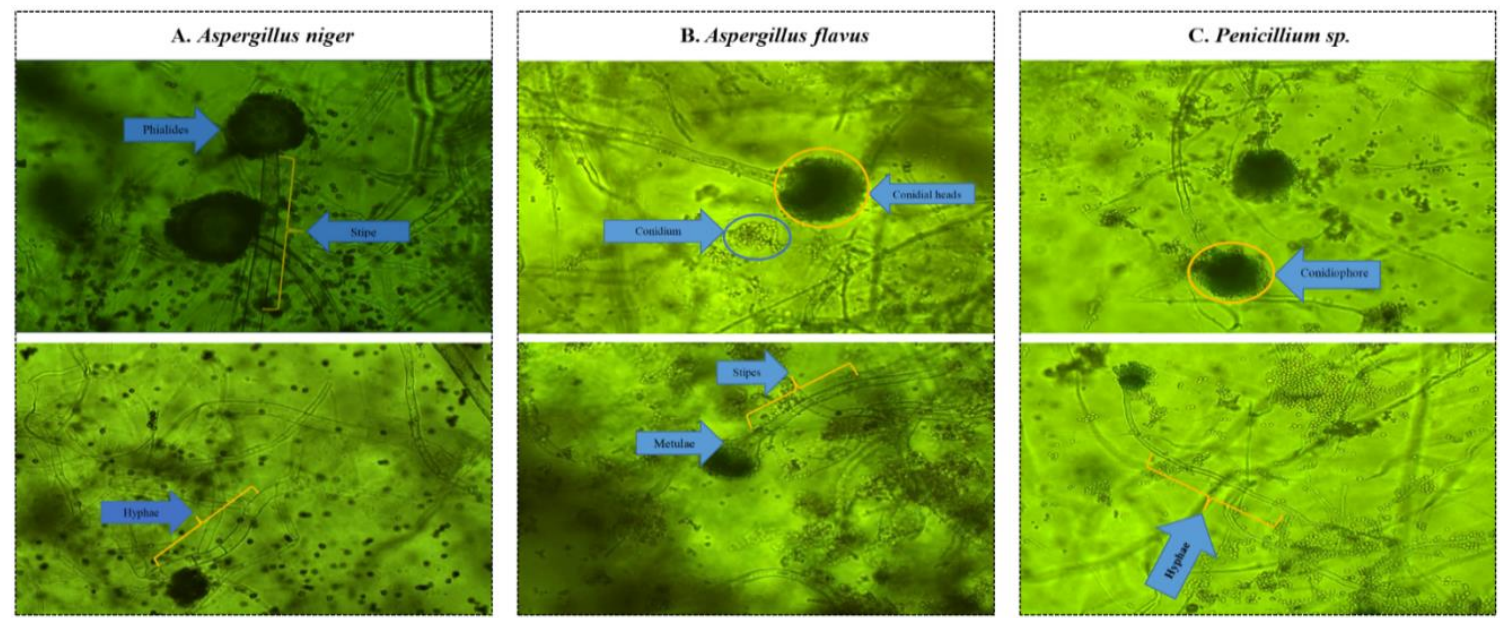

Figure 5. Microscopic features were observed under inverted microscope in 40X zoom. A. Aspergillus niger; Conidial heads were biserite with phialides and septate hyphae were observed. B. Aspergillus flavus; Conidial heads were radiate and conidiophore stipes were hyaline. C. Penicillium sp.; Hyaline hyphae and conidiophore subglobuse.

Table 2. Isolation of mycoflora from the collected samples

\begin{tabular}{|c|c|c|c|}
\hline Sample & A. niger & A. flavus & Penicillium sp. \\
\hline S1 & + & - & + \\
\hline S2 & + & + & + \\
\hline S3 & + & + & - \\
\hline S4 & + & - & + \\
\hline S5 & + & - & + \\
\hline S6 & - & - & - \\
\hline S7 & + & + & - \\
\hline S8 & + & - & - \\
\hline S9 & + & + & - \\
\hline S10 & + & - & - \\
\hline S11 & + & - & + \\
\hline S12 & + & + & - \\
\hline S13 & + & - & - \\
\hline S14 & + & - & + \\
\hline S15 & + & - & - \\
\hline
\end{tabular}




\section{Biochemical characterization for extracellular enzyme activity}

Biochemical tests were done for all fungal isolates to determine their ability to produce extracellular enzymes such as amylase, protease, lipase, and cellulase. It was found that all fungal isolates have a significant ability to produce extracellular amylase, protease, and cellulose activity (Figure 6). The fungal isolates cultured on minimal media supplemented with $1 \%$ starch followed by flooding with $1 \%$ iodine reagent to confirm the extracellular amylase activity showed positive amylase activity by forming a dark blue color zone around the fungal colony. The fungal isolates subjected for plate assay method in minimal media supplemented with $1 \%$ gelatin to assess the level of extracellular protease activity showed a clear zone around the fungal colony indicating the presence of extracellular protease activity. However, no interpretable results of lipase activity were observed in petri plates containing tween 80 agar media (Figure 6). To determine the cellulase activity of the fungal isolates, they were inoculated in media containing cellulose (Carboxymethyl cellulose) as substrate followed by staining with $1 \%$ congo red dye solution. After that, plates were destained with $1 \mathrm{M} \mathrm{NaCl}$ solution. A clear zone was formed around the colony which indicates the production of cellulase. To further increase the contrast, plates were flooded with $5 \%$ acetic acid solution and a pink color zone was formed around the fungal colony. All the fungal isolates showed cellulase activity (Figure 6).

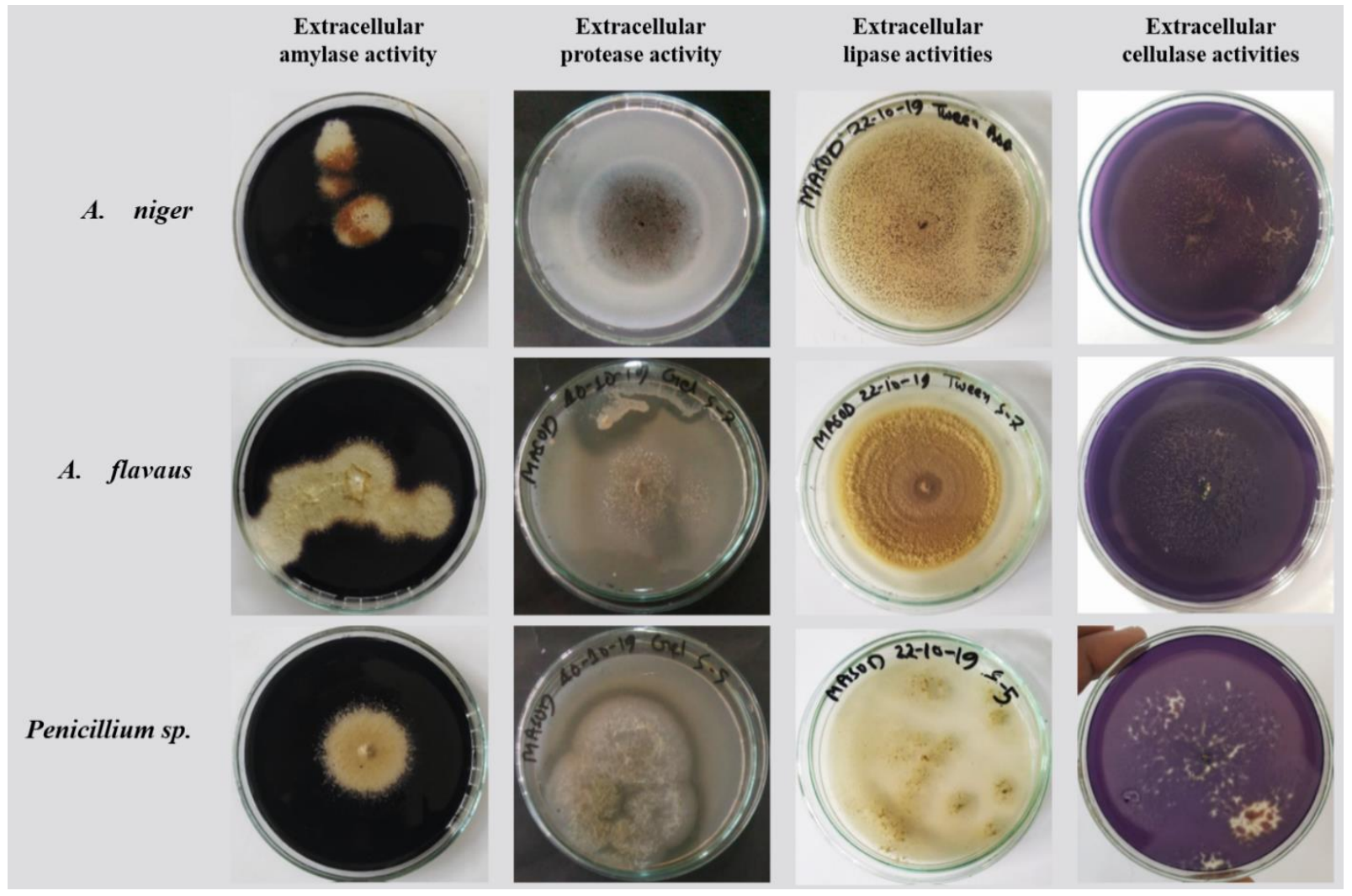

Figure 6. Extracellular enzyme activity of isolated fungal species. The four columns are serially showing extracellular amylase activity, extracellular protease activity, extracellular lipase activity, and extracellular cellulase activity, respectively. The three rows represent different fungal species, that are: Aspergillus niger, Aspergillus flavus, and Penicillium sp., respectively.

\section{Discussion}

Food security is one of the main issues in third world countries like Bangladesh. Here access to safe and sufficient food is still a challenge. Prevalence of moderate and severe food insecurity in the total population was above $30 \%$ from 2018 to 2020 in Bangladesh ${ }^{11}$. In recent years, Bangladesh has done so much in this aspect and has become synonymous in achieving food security in the case of rice, the staple food of Bangladesh. Yet food safety has not got enough attention to the policy makers. Consequently, many people in Bangladesh suffer from food-borne illnesses frequently ${ }^{19,20}$.

We found the presence of fungi in 14 out of 15 collected onion samples in our experiment. Based on the macroscopic and microscopic features of the fungi isolated from the samples,
14 samples were infected with $A$. niger whereas five and six samples were infected with A. flavus and Penicillium sp. respectively. Aspergillus species are notoriously known as the pollutant of environment, food commodities and the causal agent of human and animal pathogenesis. Opportunistic infections, allergic reaction and mycotoxicoses occur in human are made by Aspergillus species. Otomycosis, pulmonary aspergillosis and pulmonary infection are reported to be caused by Aspergillus niger and Aspergillus flavus respectively. In addition, although Penicillium spp. is well known for antibiotic production, it also causes diseases known as penicilliosis and produces a number of toxins. These filamentous fungi conidia and their associated toxinsmay enter into our body mostly through inhalation during handling, processing/cutting of the onion and off course through 
ingestion either raw or cooked form ${ }^{21}$. However, in our survey study, we found that $78 \%$ retailers do not have knowledge about fungal food spoilage, only $8 \%$ knows about fungal disease and unfortunately $100 \%$ of the retailer does not know anything about mycotoxins. On the other hand, $61 \%$ consumers know about fungal food (onion) spoilage and only 23\% knows about mycotoxins. Moreover, 94\% retailer and $54 \%$ consumers believe that dry heat or sun burn can remove fungal spore and toxins from onion completely where as $100 \%$ retailer and $78 \%$ consumers believe that cooking can remove all the contaminants from food.64\% consumers also believe that washing can make onion safe for them. From this observation, it is clear that people have some serious misconception and ignorance about fungal food contamination, fungi associated mycoses and mycotoxicoses. Because fungal infected onion may pollute their indoor environment, they may inhale spore and toxin unconsciously. Nevertheless, some of the mycotoxins arevividly thermostable and even after cooking some residue may remain in the food and may cause a number systemic diseases, organ failure and even death ${ }^{7}$.Our results are similar to the reports where survey study showed that the majority of the respondents were not informed about mold toxins and their thermal stability which leads to consumption of moldy fruits ${ }^{22,23}$. This signifies the fact that, despite being concerned about food safety, people still have a lack of knowledge about the health issues related to fungal contamination and lack proper guidance and practice regardless the age, sex, financial status, education and so on. Thus, it is urgent to create awareness about fungal spoilage of food among people from all economic classes and social status. From this study, it is evident that a wide-scale food safety regulatory framework is needed right at this moment where all stakeholders, food producers, consumers have to be brought under the same umbrella. Mass training about safe food practice and personal hygiene are much needed to make sure every individual is aware of the outcomes of unsafe food. Biochemical tests were done for all fungal isolates to determine their ability to produce extracellular enzymes such as amylase, protease, lipase, and cellulase. It was found that all fungal isolates have a significant ability to produce extracellular amylase, protease, and cellulase. No interpretable level of activity was found for lipase activity. It may be because onions contain very low lipid content. It is to say that, as the amount of lipids is very low, fungal isolates did not have to degrade lipids to infect onion samples. Interestingly, fungal amylases, proteases and especially cellulases have so many industrial applications as they have some good features such as low production cost, greater stability (thermostable and can act on a wide $\mathrm{pH}$ range), and wider availability. Isolated fungal species of our study can be a potential source of amylase, protease, and cellulase ${ }^{24-26}$.

Last but not the least, fungal research area is somewhat neglected worldwide and Bangladesh is no exception. However, this arena of research should be taken as a prioritized area as fungi are the direct threat to our food security, safety and human and animal health. Fungi related complications are much neglected worldwide, probably first, because people who are suffering from mycosis and mycotoxicosis are poorer in the society, secondly fungi associated health problem is rarely acute rather chronic.
Whatever the reasons, fungal food spoilage is a severe problem to the developing countries like Bangladesh.

\section{Conclusion}

In conclusion, it could be stated from this study that food safety in terms of fungal spoilage is a problematic issue in countries like Bangladesh. Some people were able to recognize fungi and some were not but they were unaware of the health hazard related to fungal contamination of food. People belonging to all socio demographics and socioeconomic conditions lack proper knowledge and guidance about food safety at a personal level. Survey data and laboratory data indicate the presence of fungi in onions. Although a prime number of retailers and consumers believe that washing, sunburn and cooking can remove all kinds of toxicity while in reality cooking cannot remove efficiently mycotoxin or heavy metals. A proper awareness program, as well as a strict regulatory body, is in demand of time to reduce economic loss and the public health burden due to fungi associated with food spoilage and toxicosis.

\section{References}

1. Teshika, J.D., Zakariyyah, A.M., Zaynab, T., Zengin, G., Rengasamy, K.R., Pandian, S.K. and Fawzi, M.M., 2019. Traditional and modern uses of onion bulb (Allium cepa L.): a systematic review. Critical reviews in food science and nutrition, 59(sup1), pp.S39-S70.

2. Kumar, V., Neeraj, S.S. and Sagar, N.A., 2015. Post harvest management of fungal diseases in onion-a review. International Journal of Current Microbiology and Applied Sciences, 4(6), pp.737-52.

3. Ji, S.H., Kim, T.K., Keum, Y.S. and Chun, S.C., 2018. The major postharvest disease of onion and its control with thymol fumigation during low-temperature storage. Mycobiology, 46(3), pp.242-253.

4. Silva, J.J., Bertoldo, R., Fungaro, M.H.P., Massi, F.P., Taniwaki, M.H., Sant'Ana, A.S. and Iamanaka, B.T., 2021. Black aspergilli in Brazilian onions: From field to market. International journal of food microbiology, 337, p.108958.

5. Almeida, F., Rodrigues, M.L. and Coelho, C., 2019. The still underestimated problem of fungal diseases worldwide. Frontiers in microbiology, 10, p.214.

6. Rodrigues, M.L. and Nosanchuk, J.D., 2020. Fungal diseases as neglected pathogens: A wake-up call to public health officials. PLoS neglected tropical diseases, 14(2), p.e0007964.

7. Reddy, K.R.N., Salleh, B., Saad, B., Abbas, H.K., Abel, C.A. and Shier, W.T., 2010. An overview of mycotoxin contamination in foods and its implications for human health. Toxin reviews, 29(1), pp.3-26.

8. Ali, N., Blaszkewicz, M., Alim, A., Hossain, K. and Degen, G.H., 2016. Urinary biomarkers of ochratoxin A and citrinin exposure in two Bangladeshi cohorts: follow-up study on regional and seasonal influences. Archives of toxicology, 90(11), pp.2683-2697.

9. Ali, N., Hossain, K., Blaszkewicz, M., Rahman, M., Mohanto, N.C., Alim, A. and Degen, G.H., 2016. Occurrence of aflatoxin M 1 in urines from rural and urban adult cohorts in Bangladesh. Archives of toxicology, 90(7), pp.1749-1755. 
10. Ali, A.N.M.A., 2013. Food safety and public health issues in Bangladesh: a regulatory concern. European Food and Feed Law Review, pp.31-40.

11. FAOSTAT, 2019. Food and Agriculture Commodity Production Data.Food Agric. Organ. Rome, Italy,

12. Mathers, N.J., Fox, N.J. and Hunn, A., 1998. Surveys and questionnaires. NHS Executive, Trent.

13. Zhang, K., Yuan-Ying, S. and Cai, L., 2013. An optimized protocol of single spore isolation for fungi. Cryptogamie, Mycologie, 34(4), pp.349-356.

14. HarriganWilkie, Laboratory Methods in Food Microbiology, 3rd Edition, Academic Press, 4th September 1998

15. Hasan, S., Ahmed, A., Purwar, A., Khan, N., Kundan, R. and Gupta, G., 2013. Production of extracellular enzymes in the entomopathogenic fungus Verticillium lecanii. Bioinformation, 9(5), p.238

16. Sarsaiya, S., Awasthi, S.K., Awasthi, M.K., Awasthi, A.K., Mishra, S. and Chen, J., 2018. The dynamic of cellulase activity of fungi inhabiting organic municipal solid waste. Bioresource technology, 251, pp.411-415.

17. Raper, K.B., Fennell, D.I., Fennel, Austwick, P.K.C., 1965. The Genus spergillus, reprint, Williams \& Wilkins, Pennsylvania State University, 20 Jul 2009

18. Geiser, D.M., Klich, M.A., Frisvad, J.C., Peterson, S.W., Varga, J. and Samson, R.A., 2007. The current status of species recognition and identification in Aspergillus. Studies in mycology, 59, pp.1-10.

19. Feroz, F., Shimizu, H., Nishioka, T., Mori, M. and Sakagami, Y., 2016. Bacterial and fungal counts of dried and semi-dried foods collected from Dhaka, Bangladesh, and their reduction methods. Biocontrol science, 21(4), pp.243-251.

20. Jahan, M., Rahman, M., Rahman, M., Sikder, T., UsonLopez, R.A., Selim, A.S.M., Saito, T. and Kurasaki, M., 2018. Microbiological safety of street-vended foods in Bangladesh. Journal of Consumer Protection and Food Safety, 13(3), pp.257-269.

21. Egbuta, M.A., Mwanza, M. and Babalola, O.O., 2017. Health risks associated with exposure to filamentous fungi. International journal of environmental research and public health, 14(7), p.719.

22. Matumba, L., Monjerezi, M., Kankwamba, H., Njoroge, S.M., Ndilowe, P., Kabuli, H., Kambewa, D. and Njapau, H., 2016. Knowledge, attitude, and practices concerning presence of molds in foods among members of the general public in Malawi. Mycotoxin research, 32(1), pp.27-36.

23. Redzwan, M., Mutalib, A., Sokhini, M. and Rahman, A., 2012. Socio-demographic and socio-economic determinants of adults' knowledge on fungal and aflatoxin contamination in the diets. Asian Pacific Journal of Tropical Biomedicine, 2(3), pp.S1835-S1841.

24. Li, D.C., Yang, Y.J. and Shen, C.Y., 1997. Protease production by the thermophilic fungus Thermomyces lanuginosus. Mycological Research, 101(1), pp.18-22.

25. Saleem, A. and Ebrahim, M.K., 2014. Production of amylase by fungi isolated from legume seeds collected in Almadinah Almunawwarah, Saudi Arabia. Journal of taibah university for science, 8(2), pp.90-97.

26. Ja'afaru, M.I., 2013. Screening of fungi isolated from environmental samples for xylanase and cellulase production. International Scholarly Research Notices, 2013. 